\title{
A199 POTENTIAL ROLE OF S100A4 IN AN IMIMUNE RESPONSE OF RHEUMATOID ARTHRITIS
}

Lucie Andrés Cerezo, ${ }^{1}$ Markéta Kuklová, ${ }^{1}$ Mariam Grigorian, ${ }^{2}$ Michel Neidhart, ${ }^{3}$ Steffen Gay, ${ }^{3}$ Jiři Vencovský, Ladislav Šenolt ${ }^{1}$ Institute of Rheumatology, Department of Experimental Rheumatology, 1st Faculty of Medicine, Charles University in Prague, Czech Republic; ${ }^{2}$ Department of Molecular Cancer Biology, Copenhagen, Denmark; ${ }^{3}$ Center of Experimental Rheumatology, University Hospital Zurich, Switzerland

10.1136/ard.2010.149021.9

Background Rheumatoid arthritis (RA) is characterised by systemic and local inflammation caused by enhanced activity of immune cells producing several inflammatory mediators. S100A4 is a protein with metastasis-promoting functions that is significantly upregulated in RA, induces expression of matrix degrading enzymes and regulates apoptosis of synovial fibroblasts. Therefore, the aim of the present study was to investigate the role of S100A4 in an immune response.

Methods Peripheral blood mononuclear cells (PBMC) were isolated from patients with RA. CD14 and CD3 cells were selected from $\mathrm{PBMC}$ using microbeads. To investigate extracellular functions, S100A4, S100A8 and S100A12 proteins (1 $\mu \mathrm{g} / \mathrm{ml}$ ) were used for in vitro experiments. The protein levels of tumour necrosis factor (TNF)- $\alpha$, interleukin (IL)- 1 and IL- 6 were assessed using ELISA. Receptor involvement was investigated using antibodies against RAGE, Toll-like receptor (TLR)-4, TLR-2 and inhibitor of MyD88. To determine intracellular signalling pathways, inhibitors of NFאB, p38, ERK1/ ERK2 and JNK were used.

Results S100A4 significantly upregulated expression and production of TNF $\alpha$, IL-1 and IL- 6 in PBMC compared to unstimulated cells $(p<0.001)$. Importantly, production of these cytokines was markedly enhanced in response to S100A4 compared to S100A8 or S100A9. In addition, S100A4 upregulated TNF $\alpha$, IL-1 and IL- 6 in both CD14 and CD3 cells $(p<0.005)$. Furthermore, S100A4 induced secretion of these cytokines was significantly blocked by NFKB inhibitor ( $<<0.01)$, MyD88 inhibitor $(p<0.02)$ and antibody against TLR-4 $(p<0.02)$, but not by antibodies against RAGE or TLR-2. Blockade of MAPkinase p38 inhibited most significantly synthesis of TNF- $\alpha$.

Conclusion This is the first study to demonstrate S100A4 as a potent proinflammatory mediator involved in an immune response. Here the authors show that S100A4 induces production of TNF- $\alpha$, IL- 1 and IL- 6 in PBMC via TLR- 4 and NFאB dependent pathway and thus may contribute to the pathogenesis of RA. 